\title{
Critical behavior of the frustrated antiferromagnetic six-state clock model on a triangular lattice
}

\author{
J. D. Noh and H. Rieger \\ Theoretische Physik, Universität des Saarlandes, 66041 Saarbrücken, Germany \\ M. Enderle, and K. Knorr \\ Technische Physik, Universität des Saarlandes, 66041 Saarbrücken, Germany
}

\begin{abstract}
We study the anti-ferromagnetic six-state clock model with nearest neighbor interactions on a triangular lattice with extensive Monte-Carlo simulations. We find clear indications of two phase transitions at two different temperatures: Below $T_{I}$ a chirality order sets in and by a thorough finite size scaling analysis of the specific heat and the chirality correlation length we show that this transition is in the Ising universality class (with a non-vanishing chirality order parameter below $T_{I}$ ). At $T_{K T}\left(<T_{I}\right)$ the spin-spin correlation length as well as the spin susceptibility diverges according to a Kosterlitz-Thouless (KT) form and spin correlations decay algebraically below $T_{K T}$. We compare our results to recent $\mathrm{x}$-ray diffraction experiments on the orientational ordering of $\mathrm{CF}_{3} \mathrm{Br}$ monolayers physisorbed on graphite. We argue that the six-state clock model describes the universal feature of the phase transition in the experimental system and that the orientational ordering belongs to the KT universality class.
\end{abstract}

\section{INTRODUCTION}

The study of frustrated two-dimensional spin models is motivated by their relevance for phase transitions in a wide range of physical systems. For instance, the fully frustrated $X Y(\mathrm{FF} X Y)$ model describes an array of Josephson junctions under an external magnetic field [1,2]. Besides its experimental relevance the FF XY model has been the focus of a number of theoretical works since a delicate question arises here regarding the critical behavior. The model has a continuous $U(1)$ symmetry and a discrete Ising, chiral or $Z_{2}$ symmetry that can be broken at low temperatures through a KosterlitzThouless (KT) type transition and an Ising-like transition, respectively.

Despite a lot of efforts [3-22], there is no consensus on the nature of the phase transitions and it is not clear whether the two transitions happen at two different temperatures or at a single one. Renormalization group (RG) studies on the FF $X Y$ model drew the conclusion that the transitions occur at the same temperature [3]. Monte Carlo simulation studies on generalized FF $X Y$ models supported the single transition picture for the FF $X Y$ model $[$. Interestingly, it has been reported that the $Z_{2}$ symmetry breaking transition may not belong to the Ising universality class. Monte Carlo studies of the FF $X Y$ models on a triangular and a square lattice yielded the correlation length exponent $\nu=0.83(4)$ (triangular lattice) and $\nu=0.85(3)$ (square lattice), which is inconsistent with the Ising value $\nu=1$ [5]. The specific heat appeared to follow a power-law scaling rather than the logarithmic scaling as one would expect for the Ising universality class. A non-Ising scaling behavior is also observed in the studies of the FFXY model via a Monte Carlo simulation [6] and Monte Carlo transfer matrix cal- culations [7,8]. The same non-Ising critical behavior is also observed in the coupled $X Y$-Ising model [5,9, 10$]$.

On the other hand, numerical evidence also in favor of two transitions at two different temperatures has been collected. Monte Carlo simulation studies of the frustrated Coulomb gas system, which is supposed to be in the same universality class as the FF $X Y$ model, showed that the KT type transition temperature $T_{K T}$ and the Ising-like transition temperature $T_{I}$ are different with $T_{K T}<T_{I}$ 11, 12. Two transitions were also found in the FF $X Y$ model on a square lattice [13] and on a triangular lattice [14 16] using Monte Carlo simulations. A careful analysis of the RG flow of the FF $X Y$ model also led to a conclusion of the double transition scenario [17]. It is the general belief that the transitions at $T_{K T}$ and at $T_{I}$ belong to the KT universality class [18] and to the Ising universality class, respectively, if the transitions occur at different temperatures. However, the critical exponents associated with the $Z_{2}$ symmetry breaking, which are found by Monte Carlo simulations, turn out to be different from those of the Ising universality 12, 13, 16. They are rather close to those obtained in the $X Y$-Ising model [5]. Although there is an argument that the observed non-Ising exponents are due to a screening effect hindering the asymptotic scaling behavior [19], the controversy on the nature of the phase transition remains unsettled 2022].

Here we present a thorough numerical study of a related model, the fully frustrated anti-ferromagnetic sixstate clock model on a triangular lattice. We also report on an orientational ordering transition in a twodimensional experimental system [23] that, as we argue, is in the same universality class as the model we study numerically. The experimental system actually motivates (besides numerical simplicity) our restriction to the six 
states of the spins rather than the continuum $X Y$ spins (and thus to a six-fold clock $\left(C_{6}\right)$ symmetry rather than the $U(1)$ symmetry). In the unfrustrated (ferromagnetic) case it has been shown, however, that the KT-behavior is stable with respect to a crystal field of six-fold symmetry [24 26]. Hence we expect that our model displays the same critical behavior as the FF $X Y$ model.

The paper is organized as follows: In Sec. II we define the model and identify symmetry in the ground state. The corresponding order parameters are also defined. In Sec. III we explain briefly the Monte-Carlo procedure we used and determine the transition temperatures for the two phase transitions. Section IV is devoted to the classification of the universal properties at the two transitions we find. In Sec. V we present experimental x-ray diffraction results on the orientational ordering of $\mathrm{CF}_{3} \mathrm{Br}$ monolayers physisorbed on graphite which we expect to be described by the KT-transition occurring in the model we studied numerically in the preceding sections. Section VI concludes the paper with a summary of our results.

\section{MODEL}

We investigate the phase transitions of the antiferromagnetic six-state clock model on a two-dimensional (2D) $N=L_{x} \times L_{y}$ triangular lattice (see Fig. 1). The six-state clock spin $\mathbf{S}$ is a planar spin pointing toward discrete six directions; $\mathbf{S}=(\cos \theta, \sin \theta)$ with

$$
\theta=\frac{2 \pi n}{6} \quad(n=0,1, \ldots, 5) .
$$

The interaction is given by the Hamiltonian

$$
\mathcal{H}=2 J \sum_{\langle i, j\rangle} \cos \left(\theta_{i}-\theta_{j}\right)
$$

where the sum is over all nearest neighbor site pairs $\langle i, j\rangle$ and $J>0$ is the antiferromagnetic coupling strength. The overall factor 2 is introduced for a computational convenience.

The antiferromagnetic interaction on a triangular lattice induces a frustration. As a result the spins on each triangles should make an angle of $\pm 120^{\circ}$ with one another in the ground state. There are 12-fold degenerate ground states with three-sublattice structure. According to their chirality, we can categorize the ground states into $\mathcal{C}_{n}$ and $\mathcal{A}_{n}(n=0,1, \ldots, 5)$. In each ground state the spin configurations of the three sublattices $A, B, C$ (Fig. 1) are given by

$$
\theta_{i \in A}=\frac{2 \pi n}{6}, \theta_{i \in B}=\frac{2 \pi(n \pm 2)}{6}, \theta_{i \in C}=\frac{2 \pi(n \pm 4)}{6}
$$

for $\mathcal{C}_{n}$ (upper sign) and $\mathcal{A}_{n}$ (bottom sign). In other words, in the $\mathcal{C}(\mathcal{A})$ type ground state, the spin angles

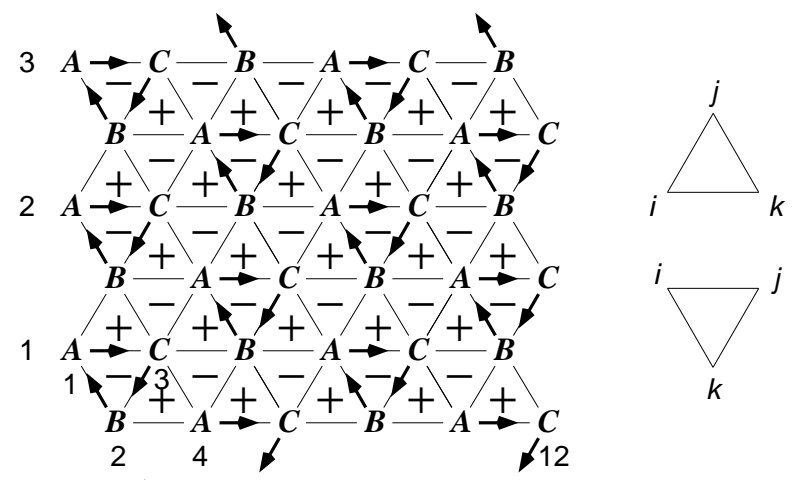

FIG. 1. A triangular lattice of size $12 \times 3$. The arrows represent the spin state in one of the ground states, $\mathcal{C}_{0}$. The lattice sites are labeled by the sublattice indices $A, B$, and $C$. Also shown are elementary up- and down- triangles denoted by $\triangle_{i}$ and $\nabla_{i}$, respectively. The \pm signs denote the chirality of the triangles.

increase by $\frac{2 \pi}{3}$ along the up-triangles in the (anti-) clockwise direction, and vice versa for down-triangles.

There are two different symmetries between them; those in the same class of $\mathcal{C}$ or $\mathcal{A}$ are related by the rotation $\theta_{i} \rightarrow \theta_{i}+\frac{2 \pi m}{6}(m=0, \ldots, 5)$ and those in the others by the reflection $\theta_{i} \rightarrow-\theta_{i}$. Therefore the model possesses the $C_{6}$ (six-fold clock) symmetry and the $Z_{2}$ (Ising) symmetry. Note that the FF $X Y$ model on a triangular lattice has a $U(1)$ and a $Z_{2}$ symmetry [2]. Since the clock spin can have only six states, while the $X Y$ spin is a continuous one, the continuous $U(1)$ symmetry of the FF $X Y$ model is reduced to the discrete $C_{6}$ symmetry. The nature of the $Z_{2}$ symmetry in each model is identical.

From the symmetry consideration, we expect that there are two types of phase transitions associated with the spontaneous breaking of the $C_{6}$ symmetry and the $Z_{2}$ symmetry. The chirality at each elementary triangle is defined as

$h_{\triangle_{i}, \nabla_{i}}=\frac{2}{3 \sqrt{3}}\left[\sin \left(\theta_{j}-\theta_{i}\right)+\sin \left(\theta_{k}-\theta_{j}\right)+\sin \left(\theta_{i}-\theta_{k}\right)\right]$,

where $\triangle_{i}$ and $\nabla_{i}$ are up- and down-triangles as depicted in Fig. 1. Then the ground state $\mathcal{C}_{n}\left(\mathcal{A}_{n}\right)$ has a checkerboard pattern of the chirality with $h_{\triangle}=+1(-1)$ and $h_{\nabla}=-1(+1)$. The staggered chirality

$$
h=\frac{1}{2 N} \sum_{i}\left(h_{\triangle_{i}}-h_{\nabla_{i}}\right)
$$

plays a role of the order parameter for the $Z_{2}$ symmetry breaking transition. The order parameter for the $C_{6}$ symmetry breaking transition is the sublattice magnetization

$$
m_{A}=\frac{1}{N} \sum_{i \in A} \exp \left(i \theta_{i}\right),
$$

where the sum runs only over the sites in the $A$ sublattice. $m_{B}$ and $m_{C}$ are defined analogously. 


\section{TRANSITION TEMPERATURES}

We performed Monte Carlo (MC) simulations on finite $N=L \times(L / 2)$ lattices with a sublattice updating scheme; one of the three sublattices is selected randomly and then all spins in the chosen sublattice are flipped according to the Metropolis rule. One Monte Carlo step corresponds to three sublattice updates. Various observables are measured during the MC runs, such as the energy, the chirality, and the sublattice magnetization, from which we can measure the averaged quantities and their fluctuations. In some cases, histograms are constructed from particularly long MC runs to obtain the observables as continuous functions of the temperature.

The transition temperature is determined from the Binder parameter

$$
B_{h}=1-\frac{\left\langle h^{4}\right\rangle}{3\left\langle h^{2}\right\rangle^{2}} \text { and } B_{m}=1-\frac{\left\langle m_{A}^{4}\right\rangle}{3\left\langle m_{A}^{2}\right\rangle^{2}}
$$

for the chirality and the magnetization, respectively. Here the angle bracket denotes a thermal average, which can be done by a time average over the MC runs. The Binder parameter is scale-independent at the critical points and approaches $\frac{2}{3}(0)$ in the ordered (disordered) phase as $L$ becomes larger. Hence the order-disorder transition temperature $T_{I}$ related to the $Z_{2}$ symmetry breaking is obtained from the crossing point in the plot of $B_{h}$ versus $T$ at different system sizes. From Fig. 2 (a), we estimate that

$$
T_{I}=1.038 \pm 0.0005
$$

$B_{m}$ also displays the crossing behavior (Fig. 2 (b)), from which we estimate that

$$
T_{K T}=1.035 \pm 0.0005
$$

for the $C_{6}$ symmetry breaking transition.

The Binder parameters show different size dependence at low temperatures. For the staggered chirality $B_{h}$ converges to $2 / 3$ rapidly as $L$ increases. However, $B_{m}$ appears to converge to values less than $2 / 3$ at $T<T_{K T}$. It indicates that there is a quasi-long-range order in spins at $T<T_{K T}$. We confirm it from finite-sizescaling (FSS) behaviors of the magnetization. The sublattice magnetization shows the power law scaling behavior, $\left\langle\left|m_{A}\right|\right\rangle \sim L^{-x}$, at $T \leq T_{K T}$ with temperature dependent exponent $x$ (see Fig. 3).

The two transition temperatures lie very close to each other. Nevertheless the accuracy of the data is sufficiently high that we can exclude a possibility $T_{I}=T_{K T}$. Figure 1 demonstrates this via the correlation functions. We measure the correlations between the chirality of uptriangles and the magnetization of spins in the $A$ sublattice displaced by a distance $r$ in the vertical direction:
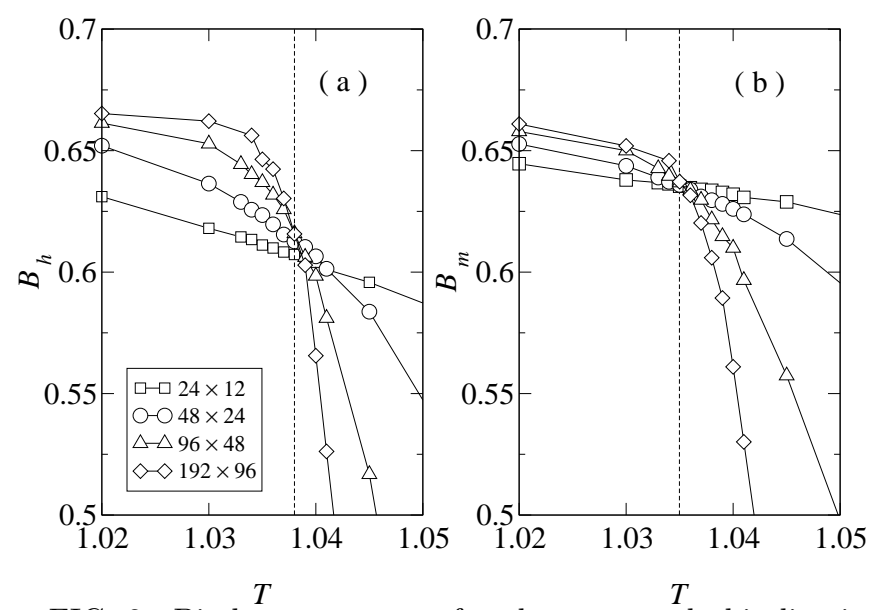

FIG. 2. Binder parameters for the staggered chirality in (a) and the magnetization in (b). The estimated transition temperatures are marked by broken lines.

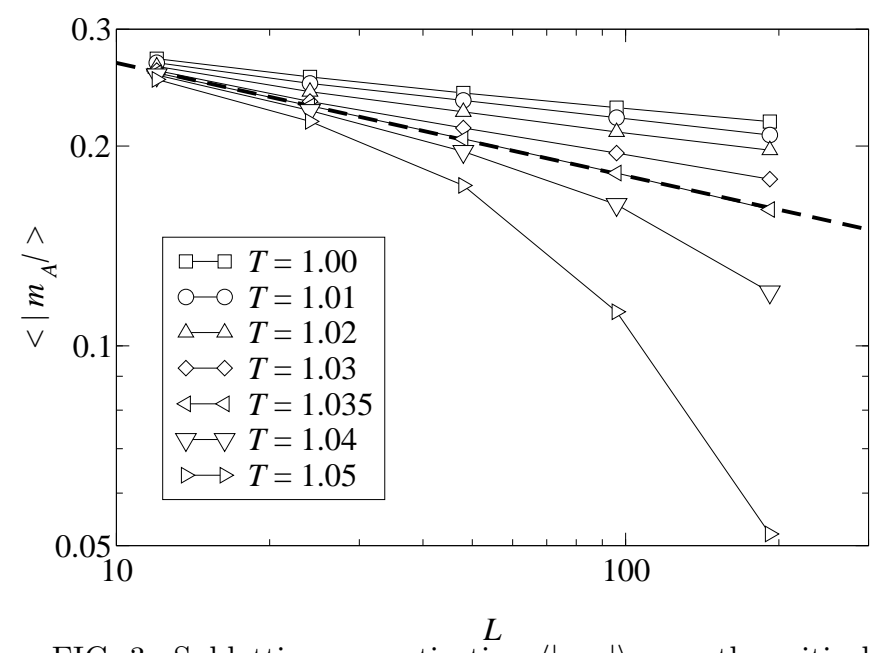

FIG. 3. Sublattice magnetization $\left\langle\left|m_{A}\right|\right\rangle$ near the critical temperature $T_{K T}$. At temperatures $T \leq T_{K T}$ it shows a power law behavior. The broken line has a slope -0.17 .

$$
\begin{gathered}
C_{h}(r)=\left\langle\frac{3}{N} \sum_{i \in A} h_{\triangle_{i}} h_{\triangle_{i+r}}\right\rangle, \\
C_{m}(r)=\left\langle\frac{3}{N} \sum_{i \in A} \cos \left(\theta_{i}-\theta_{i+r}\right)\right\rangle .
\end{gathered}
$$

Here, $i+r$ denotes a site displaced vertically by a distance $r$ from $i$, and $\triangle_{i}$ denotes an up-triangle whose left corner is $i$ (see Fig. 1). In Fig. 囵, we plot both correlation functions in the log-log scale at an intermediate temperature $T=1.037$. Clearly one can see an upward curvature in the plot of $C_{h}(r)$ for $r \ll L_{y} / 2$, which implies that the chirality order has already set in. On the other hand, there is a downward curvature in the plot of $C_{m}(r)$ for $r \ll L_{y} / 2$ indicating that the magnetic order has not set in yet. Therefore we conclude that $T_{K T}<1.037<T_{I}$. 

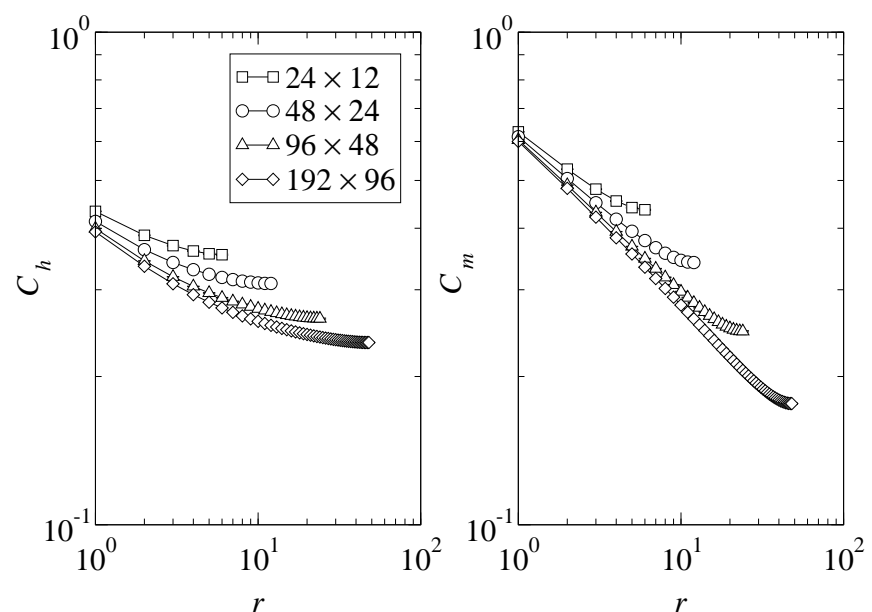

FIG. 4. Correlation functions of the staggered chirality, $C_{h}$, and the magnetization, $C_{m}$, at temperature $T=1.037$.

\section{UNIVERSALITY CLASS}

With $T_{K T} \neq T_{I}$, one expects that the both symmetry breakings take place independently. Then, the $Z_{2}$ symmetry breaking transition should be in the Ising universality class. The $C_{6}$ symmetry is equivalent to that of the $X Y$ model perturbed by the $(p=6)$-fold anisotropy field. The unperturbed $X Y$ model displays a KT transition which separates a disordered high-temperature phase and a quasi-long range ordered low-temperature phase. A renormalization group (RG) study shows that an anisotropy field with $p>4$ does not change the KT nature of the transition [24]. The same is true for the extreme case of the ferromagnetic six-state clock model [26]. So the $C_{6}$ symmetry breaking phase transition at $T=T_{K T}$ is expected to belong to the KT universality class.

The interplay of the Ising type and the KT type ordering has long been studied in the context of the fully frustrated $X Y$ models on a square or a triangular lattice. Frequently, it has been claimed [3 8] that the Ising-like transition and the KT-type transition occur at the same temperature. On the other hand, more recent studies have reported the transitions to occur at two different temperatures $[11,[17,19,20,22]$. Surprisingly, some of recent high-precision Monte Carlo simulation studies suggested that the $Z_{2}$ symmetry breaking transition does not belong to the Ising universality class in spite of the double transition 13, 16,22. They estimated that $\nu \simeq 0.8$ for the correlation length exponent and $\alpha / \nu \simeq 0.46$ with the specific exponent $\alpha$, which are incompatible with the Ising values of $\nu=1$ and $\alpha=0$. The critical exponents are consistent with the values observed along the single transition line of the coupled $X Y$-Ising model [5, 9, 10]. Olsson [19] argued that the measured non-Ising critical exponents are artifacts of unusual finite size effects originated from non-critical spin-wave fluctuations. However, apparently there is no general agreement yet on this sub- ject [20].

In our model, we observe the phase transitions occurring at two different temperatures. However, the controversy existing in the FF $X Y$ model tells us that it does not guarantee necessarily that the individual transitions will belong to the KT universality class and the Ising universality class, respectively. Therefore we perform a thorough finite-size-scaling (FSS) analysis to understand the nature of the phase transition. We believe that the antiferromagnetic six-state clock model can be used to resolve the existing controversy for the FF $X Y$ model. It has the proper symmetry properties as discussed previously. And, from a practical point of view, larger system sizes are available since it is a discrete model, with which one can reduce the finite size effects.

\section{A. $Z_{2}$ symmetry breaking}

We investigate the nature of the $Z_{2}$ symmetry breaking transition by exploring the FSS property of the specific heat, defined by

$$
c=N\left[\left\langle e^{2}\right\rangle-\langle e\rangle^{2}\right]
$$

with $e$ the energy per site. For a finite system $(N=$ $L \times L / 2)$ the specific heat has a peak $c_{*} \sim L^{\alpha / \nu}$ near the critical temperature, diverging with system size $L \rightarrow$ $\infty$. Since the specific heat does not diverge at a KT type transition, the divergence is due to the $Z_{2}$ symmetry breaking phase transition. The Ising universality class has $\alpha=0$, and the specific heat shows a logarithmic divergence, $c_{*} \sim \ln L$.

The specific heat is measured accurately using the standard Monte Carlo (MC) method combined with a single histogram method [27]. First we measure the specific heat from the standard MC simulations at discrete temperature grid, which leads to a temperature $T_{0}$ at which the specific heat is maximum approximately. Then the histogram $\Omega_{0}(\{\mathbf{S}\})$, frequency of a spin state $\{\mathbf{S}\}$, is constructed from MC runs at the temperature $T_{0}$ over a time interval $\Delta t$. It enables us to obtain the average value of any observable $Q$ as a continuous function of temperature $T$ near $T_{0}$;

$$
\langle Q\rangle=\frac{\sum_{\{\mathbf{S}\}} Q(\{\mathbf{S}\}) \Omega_{0}(\{\mathbf{S}\}) e^{-\left(T^{-1}-T_{0}^{-1}\right) E(\{\mathbf{S}\})}}{\sum_{\{\mathbf{S}\}} \Omega_{0}(\{\mathbf{S}\}) e^{-\left(T^{-1}-T_{0}^{-1}\right) E(\{\mathbf{S}\})}} .
$$

It is important to take $\Delta t$ as large as possible to have good statistics. At $192 \times 96$ lattice, for example, we take $\Delta t=5 \times 10^{7}$ which is $\sim 2000$ times of the relaxation time of energy-energy auto-correlation. The maximum system size we can simulate is $384 \times 192$. In that case we take $\Delta t=10^{8}$ which is $\sim 1200$ times of the relaxation time of energy-energy auto-correlation. Figure 5 shows the specific heat obtained from independent MC runs and 

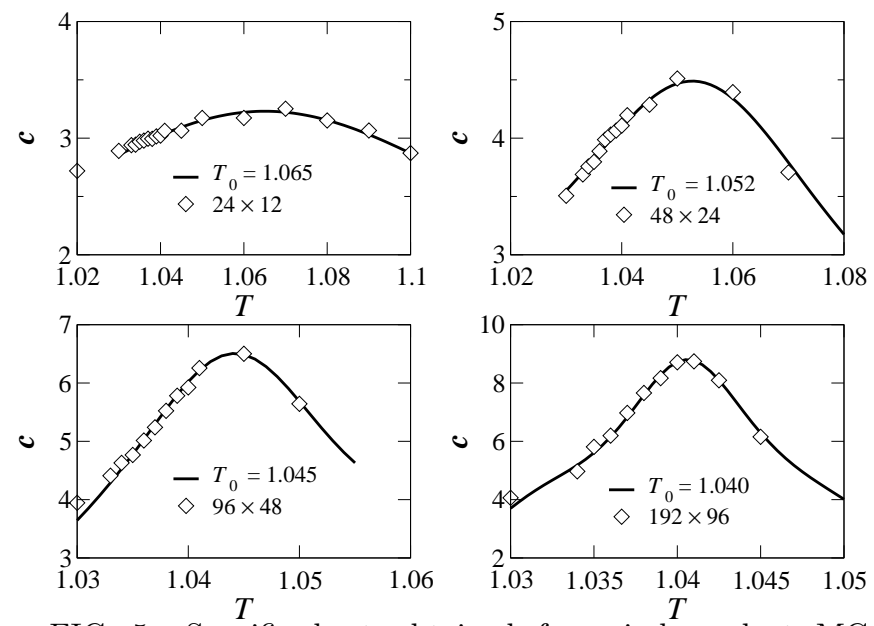

FIG. 5. Specific heat obtained from independent $\mathrm{MC}$ runs (symbol) and the histogram method (solid lines). $T_{0}$ denotes the temperature at which the histogram is obtained.

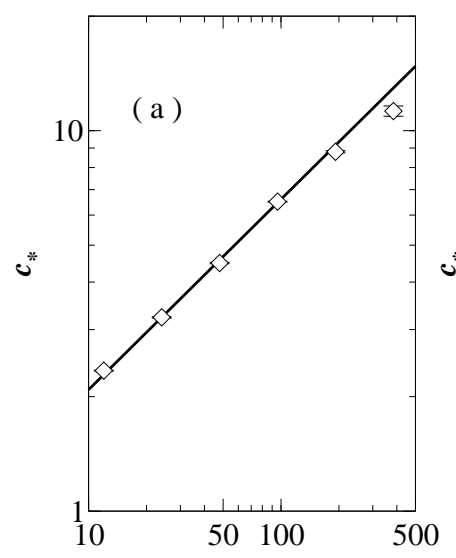

$L$

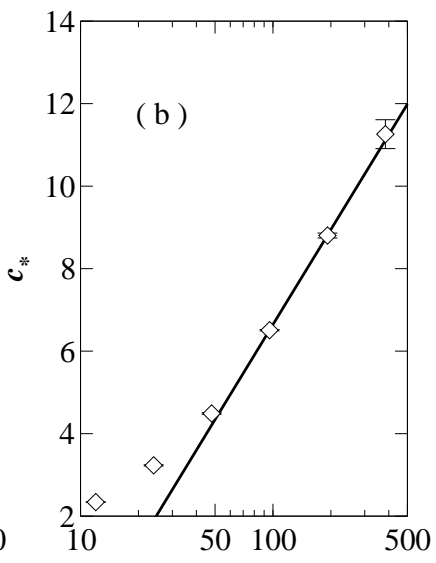

$L$
FIG. 6. Peak values of the specific heat versus $L$ in log-log scale (a) and semi-log scale (b). In (a) the solid line has a slope 0.5 .

from the histogram method. Their agreement near the peak is excellent, although they begin to deviate slightly away from the peak. With this technique we can estimate $c_{*}$ very accurately. A statistical error for $c_{*}$ is estimated in the following way; we construct ten sub-histograms and as many values of $c_{*}$ from each of them. The error bar is taken as the half distance between the maximum and minimum values among them.

The result is shown in Fig. 6. Interestingly, there is a crossover from a power-law scaling behavior $c_{*} \sim L^{0.5}$ (solid line in Fig. 6(a)) at $L \leq L_{c}$ to a logarithmic scaling behavior $c_{*} \sim \ln L$ (solid line in Fig. $6\left(\right.$ (b)) at $L \geq L_{c}$ with $L_{c} \simeq 96$. It indicates that the specific heat has a logarithmic scaling behavior asymptotically, which is consistent with the Ising universality class.

We also study the scaling of the chirality correlation length. We measured the correlation length using $\xi_{h}=\sqrt{\sum_{r} r^{2} C_{h}(r) / \sum_{r} C_{h}(r)}$ with the correlation function defined in Eq. (9). It is plotted in Fig. 17. We find

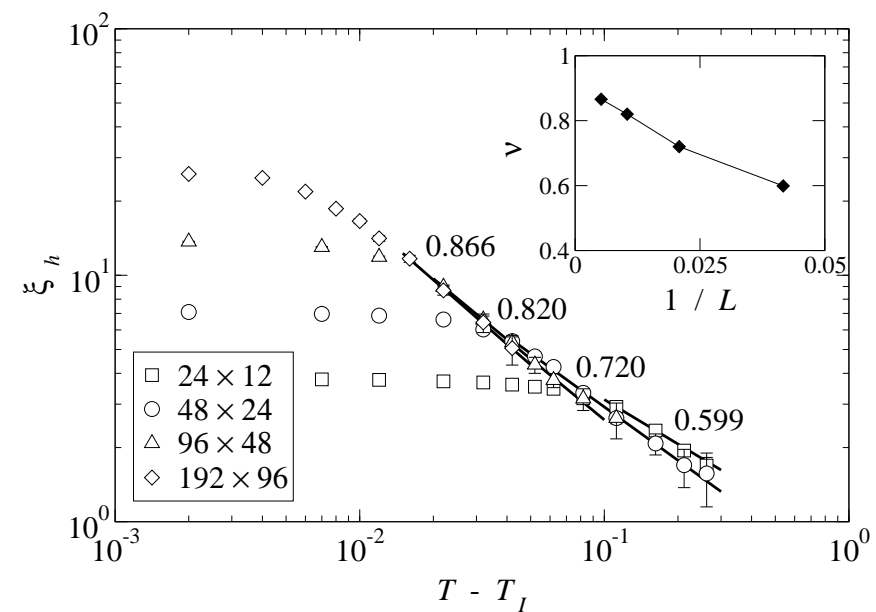

FIG. 7. Chirality correlation length. The values of the exponent $\nu$ obtained from the fit at each lattice size are shown in the main panel and plotted in the inset.

a strong dependence of $\xi_{h}$ on the system size. In addition to the saturation of $\xi_{h}$ to $O(L)$ near and below the critical point, the dependence of the correlation length on $L$ in the regime $\xi_{h} \ll L$ is visible through a varying slope (cf., Fig. G). As a consequence, a fitting to a form $\xi_{h}=a\left(T-T_{I}\right)^{-\nu}$ at small system size will lead to a lower value of $\nu$ than its asymptotic value. The fitted values of $\nu$ at each lattice size are plotted in the inset of Fig. 7 . It increases as the system size and approaches the Ising value $\nu \simeq 1.0$ in the infinite size limit.

From the analysis of the specific heat and the chirality correlation length, we conclude that the $Z_{2}$ symmetry breaking transition indeed belongs to the Ising universality class. Note that the effective specific heat exponent $\alpha / \nu \simeq 0.5$ and the correlation length exponent $\nu \simeq 0.8$ appearing at small length scale are comparable with the results of previous $\mathrm{MC}$ works claiming the non-Ising nature in the frustrated $X Y$ models $[5,6,9,10,13,16,21,22]$. Our results are fully consistent with Olsson's argument claiming that the non-Ising exponents reported by others are explained by a failure of finite-size-scaling at small length scale due to the screening length associated with the nearby KT transition [19]. The strong finite size effect is overcome in this work since we could study large systems. We expect that one could observe the same crossover to the asymptotic Ising type scaling behavior in larger scale simulations in the frustrated $X Y$ systems.

\section{B. $C_{6}$ symmetry breaking}

The KT transition is characterized by the essential singularity of the correlation length and the susceptibility approaching the critical temperature. We measure the correlation length $\xi_{m}$ as 

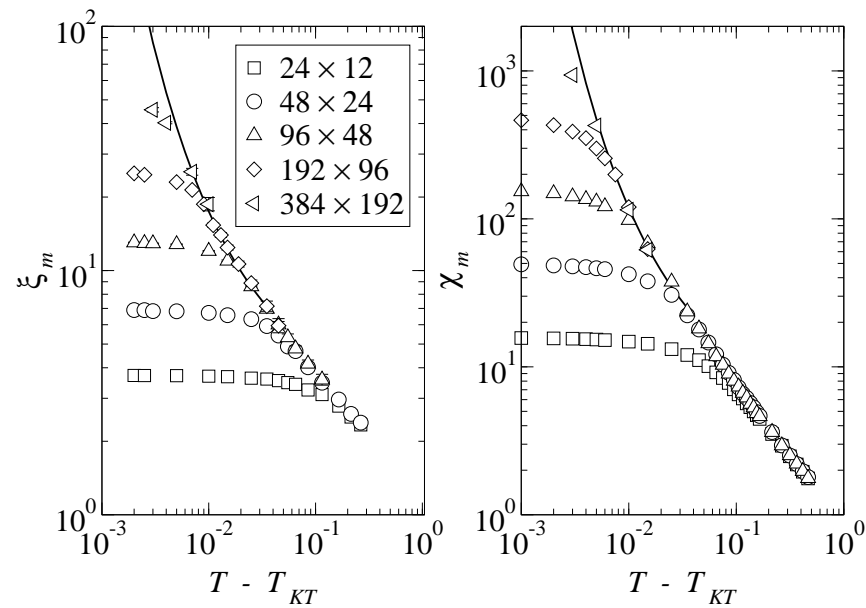

FIG. 8. Correlation length and the susceptibility of the magnetization. The solid lines are the fits to the KT form of $\xi_{m}=2.54 e^{0.193\left(T-T_{K T}\right)^{-1 / 2}}$ and $\chi_{m}=4.20 e^{0.333\left(T-T_{K T}\right)^{-1 / 2}}$.

$$
\xi_{m}=\sqrt{\frac{\sum_{r} r^{2} C_{m}(r)}{\sum_{r} C_{m}(r)}}
$$

from the magnetic correlation function $C_{m}$ in Eq. (10). One can also estimate the correlation length by fitting $C_{m}(r)$ to a form with $e^{-r / \xi_{m}}$. We tried with various function forms but found that Eq. (13) yields the estimate most stable against statistical errors. The magnetic susceptibility is measured from the fluctuation of the magnetization, $\chi_{m}=N\left\langle m_{A}^{2}\right\rangle$. Figure 8 shows the plot of $\xi_{m}$ and $\chi_{m}$ versus $T-T_{K T}$ in log-log scale. Upward curvature in both plots indicates a divergence stronger than algebraic. We fit those quantities with the KT scaling form, $\xi_{m}=a e^{b\left(T-T_{K T}\right)^{-y}}$ and $\chi_{m}=a^{\prime} e^{b^{\prime}\left(T-T_{K T}\right)^{-y}}$ with $y=\frac{1}{2}$ fixed, whose results are drawn with solid lines in Fig. 8. It shows that the KT scaling behaviors are emerging only after $L \geq 96$. If $y$ is also taken as a fitting parameter, one obtains $y<0.5$ whose value depends on the fitting range; it increases as approaching the critical temperature.

In the $X Y$ phase $\left(T<T_{K T}\right)$ the spins have the quasilong range order; the magnetization scales algebraically as $\left\langle\left|m_{A}\right|\right\rangle \sim L^{-x}$ with temperature dependent exponent $x$ (see Fig. 3). The RG theory predicts that $x=1 / 8$ at the KT transition point [24]. Our numerical data show that $x \simeq 0.17$ at $T=T_{K T}$. We think that the discrepancy is originated from a sensitive dependence of $x$ on $T$. In summary, in spite of a quantitative disagreement, the $C_{6}$ symmetry breaking phase transition is in qualitative agreement with the KT universality class; we observe the essential singularity in the correlation length and the susceptibility and quasi-long range order in the low temperature phase.

\section{EXPERIMENTAL REALIZATION: CF $_{3}$ BR ON GRAPHITE}

In this section we describe a possible experimental realization of the theoretical model we investigated above. In a recent work we reported results on $\mathrm{x}$-ray powder diffraction study on a monolayer of halomethane $\mathrm{CF}_{3} \mathrm{Br}$ adsorbed on exfoliated graphite 23. $\mathrm{CF}_{3} \mathrm{Br}$ is a prolate molecule and has a dipole moment of about $0.5 \mathrm{D}$. The coverage $\rho$, temperature $T$ phase diagram is rather complex [28,29]. In [23] we concentrated on a coverage which is representative of the extended monolayer regime in which the monolayer lattice is commensurate with the graphite lattice. This yields a $2 \times 2$ triangular lattice arrangement of the $\mathrm{CF}_{3} \mathrm{Br}$ molecules below a temperature of $105 \mathrm{~K}$ [30], which is the melting temperature of the commensurate layer. The inter-molecular distance is $a=4.92 \AA$. Note that the lateral size of the graphite crystallites is only around $180 \AA$, which confines any spatial correlation length to this value.

An isolated $\mathrm{CF}_{3} \mathrm{Br}$ would prefer to lie flat on the substrate, but the $2 \times 2$ mesh is too tight to accommodate the molecules in this orientation. Therefore the individual $\mathrm{CF}_{3} \mathrm{Br}$ molecules stand on the substrate, presumably with the $\mathrm{F}_{3}$ tripod down, with a maximum tilt angles of the molecular axis up to $30^{\circ}$ with respect to the substrate normal due to steric repulsion. A tilt leads to a non-zero in-plane component of the dipole moment. We regard this component as planar pseudospin $\mathbf{S}_{i}=\left(\cos \theta_{i}, \sin \theta_{i}\right)$ with the azimuthal angle $\theta_{i}$ of the molecule. In this sense the $2 \times 2$ state is disordered with a zero time average of every $\mathbf{S}_{i}$, and is stabilized at higher temperatures by a libration and/or a precession of the molecular axis about the substrate normal.

As the temperature is decreased additional features develop in the diffraction pattern which finally, below $40 \mathrm{~K}$, can be identified [23] as Bragg peaks (with a finite width of around $(180 \AA)^{-1}$ due to the lateral size of the crystallites) indicating an orientational order in the dipole moments identical to the one depicted in Fig. 1. The temperature dependence of the correlation length $\xi$, determined from the intrinsic width of this peak, can be fitted with the KT-expression

$$
\xi=A \exp \left(B\left(T / T_{K T}-1\right)^{-1 / 2}\right)
$$

to the data for $T>40 \mathrm{~K}$ (see Fig. 3 of Ref. [23]). The fit parameters are $A=9 \pm 2 \AA, B=1.5 \pm 0.4, T_{K T}=$ $30 \pm 3 \mathrm{~K}$. Note that the value of $\mathrm{A}$ is reasonably close to the lattice parameter of the $2 \mathrm{D}$ mesh. Thus $\xi$ is expected to diverge at a KT-critical temperature $T_{K T}$ of about 30 $\mathrm{K}$, but the growth of the correlated regions is interrupted when $\xi$ reaches the size of the graphite crystallites. This happens at about $40 \mathrm{~K}$.

We think that the model (2) is a good description of the orientational ordering process described in this physical 


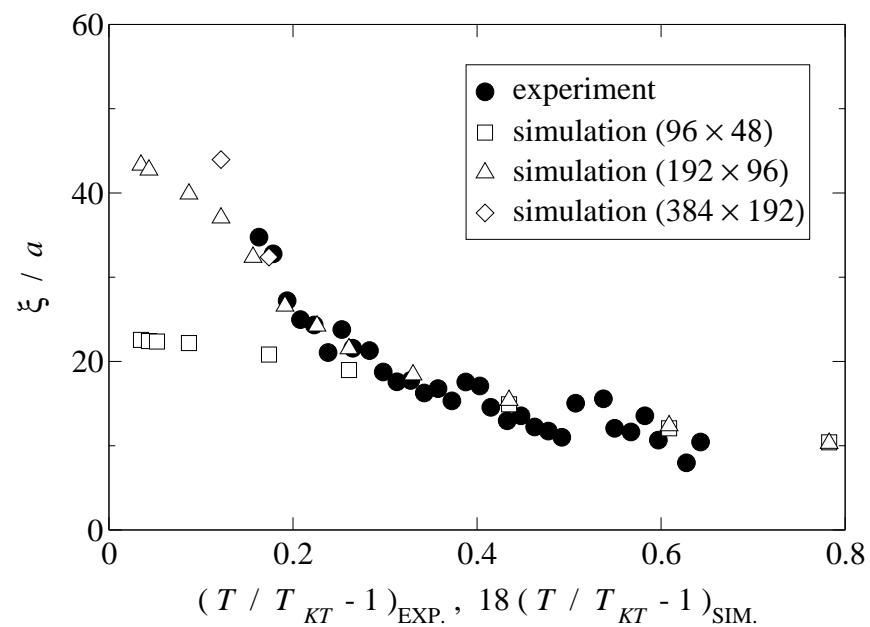

FIG. 9. Comparison of the magnetic correlation length (scaled by the lattice constant) from the experimental data (from Fig. 3 of Ref. [23]) and the Monte Carlo simulations (from Fig. 8). The reduced temperature $\left(T / T_{K T}-1\right)$ for the simulation data are rescaled by a factor of 18 in order to achieve an acceptable data collapse for linear lattice sizes $L$ between 96 and 192 .

system: Clearly the pseudospin correlations are bound to a plane, thus the system is $2 \mathrm{D}$ with respect to the relevant degrees of freedom at the phase transition. Moreover, as mentioned before, below $105 \mathrm{~K}$ the $\mathrm{CF}_{3} \mathrm{Br}$ molecules are arranged in a triangular lattice. The pseudospin representing the $\mathrm{CF}_{3} \mathrm{Br}$ dipole moment is presumably not a strictly isotropic planar rotator but experiences a crystal field from the graphite substrate which breaks the continuous azimuthal symmetry into the six fold symmetry of the monolayer. This is reflected by the six-state clock variables of model (2). The ordered structure of the $\mathrm{CF}_{3} \mathrm{Br}$ dipoles is antiferroelectric, which is taken into account by the antiferromagnetic couplings between the pseudospins in (2). Finally, the character of the relevant orientation dependent interactions of $\mathrm{CF}_{3} \mathrm{Br}$ is not known, but a comparison of monolayers of several polar methane derivatives [29] shows that fully halogenated molecules including $\mathrm{CF}_{3} \mathrm{Br}$ with small dipole moments around $0.5 \mathrm{D}$ have structures different from partially halogenated molecules such as $\mathrm{CH}_{3} \mathrm{Cl}$ with strong dipole moments around 1.7 D. This suggests that for $\mathrm{CF}_{3} \mathrm{Br}$ the short range anisotropic part of the intermolecular van der Waals force and hard-core repulsion are more important than the medium range dipole-dipole interaction. Thus the interactions can be assumed to be short ranged. Thus one expects that model (2) and the physical system discussed here are in the same universality class.

In Fig. 9 a comparison of the magnetic correlation length from the experiment [23] and the simulation (from Fig. (8) is shown. Since the factor $B$ in the KT-form (14) of the correlation length is a non-universal number the reduced temperature $\left(T / T_{K T}-1\right)$ has to be rescaled by an appropriate factor in order to achieve an acceptable data collapse. The rescaling factor turns out to be quite large, namely 18 , which is not unusual for microscopically different systems in the KT universality class (see e.g., [31]). Note that the finite linear size of the crystallites plays a similar role as the finite lattice sizes in the simulations and sets the saturation value for the correlation length (divided by the lattice constant for the triangular lattice of the $\mathrm{CF}_{3} \mathrm{Br}$ molecules, which is $a=4.92 \AA$ ). The nice collapse of the experiment and simulation data supports our claim that the two physical systems are in the same universality class and that the orientational ordering of $\mathrm{CF}_{3} \mathrm{Br}$ molecules on graphite is in the $\mathrm{KT}$ universality class.

\section{SUMMARY}

To summarize we have studied the phase transitions in the anti-ferromagnetic six-state clock model on a triangular lattice, which is fully frustrated. As a result the ground states have a $C_{6}$ (six-state clock) symmetry and a $Z_{2}$ (Ising) symmetry. Through extensive Monte Carlo simulations we found that the model undergoes a Kosterlitz-Thouless transition at $T_{K T}$ and an Ising transition at $T_{I}$. The two transitions correspond to the $C_{6}$ and the $Z_{2}$ symmetry breaking transition, respectively. High-precision Monte Carlo data indicate that the two transitions take place at different temperatures, $T_{K T}<T_{I}$ (Eqs. (7) and (8)). This has been checked explicitly by analyzing the behavior of the spin and the chirality correlation function at temperatures between $T_{K T}$ and $T_{I}$ (Fig. (4). Furthermore, we have shown that the $Z_{2}$ symmetry breaking transition belongs to the Ising universality class. For small system sizes, the scaling property of the specific heat and the correlation length deviates apparently from the Ising universality class. However, simulation results for larger system sizes indicate that the model belongs asymptotically to the Ising universality class (Figs. 6 and 7 ). As for the transition at $T_{K T}$, we have found that the magnetization correlation length and the susceptibility diverges at $T=T_{K T}$ according to the KT scaling form (Fig. 8). We have also found that the spins have a quasi long range order below $T_{K T}$ (Fig. 3). Combining these, we conclude that the transition at $T_{K T}$ belongs to the KT universality class.

Our model is a variant of the fully frustrated $X Y$ models where the KT type ordering and the Ising type ordering interplay interestingly. Our numerical results support a scenario that there are two separate phase transitions with $T_{K T} \neq T_{I}$; one at $T_{K T}$ in the KT universality class and the other at $T_{I}$ in the Ising universality class. Our results are consistent with those in Ref. [19] very well.

We have proposed that our theoretical model describes the orientational ordering transition of $\mathrm{CF}_{3} \mathrm{Br}$ molecules on graphite since the model has the same symmetry as 
the experimental system. We argue that the orientational ordering transition belongs to the KT universality class [23] by comparing the magnetic correlation length obtained from the experiment 23] with the correlation length obtained numerically in the six-state clock model. With a suitable rescaling of parameters, we have shown that the correlation lengths in both systems have the same scaling behavior (Fig.9). It gives more evidence that the orientational ordering transition is indeed the KT transition.

[1] S. Teitel and C. Jayaprakash, Phys. Rev. B 27, 598 (1983).

[2] D.H. Lee, J.D. Joannopoulos, J.W. Negele, D.P. Landau, Phys. Rev. Lett. 52, 433 (1984); Phys. Rev. B 33, 450 (1986).

[3] M.Y. Choi and D. Stroud, Phys. Rev. B 32, 5773 (1985); M. Yosefin and E. Domany, Phys. Rev. B 32, 1778 (1985).

[4] B. Berge, H.T. Diep, A. Ghazali, and P. Lallemand, Phys. Rev. B 34, 3177 (1986); H. Eikmans, J.E. van Himbergen, H.J.F. Knops, and J.M. Thijssen, Phys. Rev. B 39, 11759 (1989).

[5] J. Lee, J. M. Kosterlitz, and E. Granato, Phys. Rev. B 43, 11531 (1991).

[6] G. Ramirez-Santiago and J.V. José, Phys. Rev. Lett. 68, 1224 (1992); Phys. Rev. B 49, 9567 (1994).

[7] E. Granato and M.P. Nightingale, Phys. Rev. B 48, 7438 (1993).

[8] Y.M.M. Knops, B. Nienhuis, H.J.F. Knops, and H.W.J. Blöte, Phys. Rev. B 50, 1061 (1994).

[9] E. Granato, J.M. Kosterlitz, J. Lee, and M.P. Nightingale, Phys. Rev. Lett. 66, 1090 (1991).

[10] M.P. Nightingale, E. Granato, and J.M. Kosterlitz, Phys. Rev. B 52, 7402 (1995).

[11] G.S. Grest, Phys. Rev. B 39, 9267 (1989).

[12] J.-R. Lee, Phys. Rev. B 49, 3317 (1994).

[13] S. Lee and K.-C. Lee, Phys. Rev. B 49, 15184 (1994).

[14] S. Miyashita and H. Shiba, J. Phys. Soc. Jap. 53, 1145 (1984).

[15] H.-J. Xu and B.W. Southern, J. Phys. A 29, L133 (1996).

[16] S. Lee and K.-C. Lee, Phys. Rev. B 57, 8472 (1998).

[17] G.S. Jeon, S.Y. Park, and M.Y. Choi, Phys. Rev. B 55, 14088 (1997).

[18] J.M. Kosterlitz and D.J. Thouless, J. Phys. C 6, 1181 (1973); J.M. Kosterlitz, ibid. 7, 1046 (1974).

[19] P. Olsson, Phys. Rev. Lett. 75, 2758 (1995).

[20] J. V. José and G. Ramirez-Santiago, Phys. Rev. Lett. 77, 4849 (1995); P. Olsson, Phys. Rev. Lett. 77, 4850 (1995).

[21] E.H. Boubcheur and H.T. Diep, Phys. Rev. B 58, 5163 (1998).

[22] D. Loison and P. Simon Phys. Rev .B 61, 6114 (2000).

[23] S. Faßbender, M. Enderle, K. Knorr, J.D. Noh, and H. Rieger, Phys. Rev. B, in press (2002).

[24] J. V. José, L. P. Kadanoff, S. Kirkpatrick, and D. R. Nelson, Phys. Rev. B 16, 1217 (1977).
[25] M. Itakura, J. Phys. Soc. Jpn. 70, 600 (2001).

[26] M.S.S. Challa and D.P. Landau, Phys. Rev. B 33, 437 (1986).

[27] A.M. Ferrenberg and R. H. Swendsen, Phys. Rev. Lett. 61, 2635 (1988).

[28] E. Maus, Ph.D. thesis, Universität Mainz, 1991.

[29] K. Knorr, Phys. Rep. 214, 113 (1992).

[30] K. Knorr, S. Faßbender, A. Warken, and D. Arndt, J. Low Temp. Phys. 111, 339 (1998).

[31] S.W. Pierson, M. Friesen, S.M. Ammirata, J.C. Hunnicutt, and LeRoy A. Gorham, Phys. Rev. B 60, 1309 (1999). 